\title{
Enquête nationale du 11 juin 2013 sur les structures d'urgences : où en sommes-nous ?
}

\author{
“One-day" French National Survey on Emergency Departments: What do we Learn?
}

\author{
J. Schmidt • C. El Khoury • P.Y. Gueugniaud \\ (C) SFMU et Lavoisier SAS 2014
}

La Direction de la recherche, des études, de l'évaluation et des statistiques (DREES) du ministère de la Santé, en partenariat avec la Société française de médecine d'urgence (SFMU) et avec la participation de Samu-Urgences de France (SUdF) et de l'Association des médecins urgentistes de France (AMUF) a organisé une enquête nationale un jour donné, le 11 juin 2013, auprès de tous les patients se présentant dans l'ensemble des 738 structures d'urgences hospitalières, générales comme pédiatriques, de France métropolitaine et des départements d'Outre-Mer.

Les objectifs fixés de cette enquête prévoyaient de disposer d'une photographie de l'activité des structures des urgences, de décrire la genèse des recours et d'analyser les difficultés éventuelles rencontrées lors de la prise en charge des usagers. Il s'agissait aussi d'actualiser les données existantes sur les urgences hospitalières fondées encore pour beaucoup sur la première enquête nationale de 2002 relative aux urgences hospitalières et dont les modalités étaient différentes (les patients non hospitalisés étaient interrogés par téléphone après leur passage aux urgences, le temps de passage était

J. Schmidt $(\square)$

Pôle Samu-Smur-Urgences, CHU de Clermont-Ferrand, 58, rue

Montalembert, F-63003 Clermont-Ferrand cedex 01, France

e-mail : jschmidt@chu-clermontferrand.fr

Faculté de médecine, Université d'Auvergne Clermont 1, 28, place Henri-Dunant, F-63000 BP 38 Clermont-Ferrand cedex 1, France

C. El Khoury

Cardiologie, urgences, USC, hôpital de Vienne, F-38200 Vienne

P.Y. Gueugniaud

Pôle « URMARS » (urgences, réanimation médicale, anesthésieréanimation, SAMU), groupement hospitalier Édouard Herriot, place d'Arsonval, F-69437 Lyon cedex 03

Université Claude Bernard - Lyon 1, Faculté de médecine et de maïeutique Lyon Sud - Charles Meyrieux, 165 chemin du Grand Revoyet, BP 12, F-69921 Oullins cedex
« déclaré » par les enquêtés et non mesuré par l'équipe soignante comme dans l'enquête de 2013) [1,2].

L'originalité de l'enquête de 2013 était de mieux comprendre l'usager et de décrire avec plus de précision le temps du passage aux urgences et le devenir des malades [3]. Ainsi les questions prenaient en compte les motivations, les démarches entreprises et les conseils reçus par les patients avant de se rendre aux urgences. L'enquête abordait aussi les modalités de prise en charge liées aux comorbidités, les temps d'attente durant leur passage aux urgences en relation avec leurs pathologies, la gestion de l'aval (temps d'attente pour l'obtention d'un lit en cas d'hospitalisation du patient, hospitalisations en réanimation, durées de séjour en unité d'hospitalisation de courte de durée, etc.). Elle était complétée par un recueil administratif sur les structures d'urgences hospitalières décrivant leur organisation en lien avec leurs ressources pour la journée et s'adressait à toutes les structures d'urgences générales et pédiatriques autorisées, au sens du décret 2006-577, et à tous les patients y ayant recours le mardi 11 juin 2013 de 8 heures du matin à 8 heures le lendemain. La collecte était réalisée par les points d'accueil d'urgences générales ou pédiatriques des structures.

Les résultats, accessibles sur le site de la DREES [4], ont livré leurs premiers verdicts $[5,6]$ :

- la base finale issue de l'enquête comprend 734 points d'accueil sur les 736 recensés, soit un taux de réponse de $99,7 \%$;

- près d'une personne sur 1000 de la population résidant en France s'est rendue aux urgences le 11 juin 2013 ;

- 52018 passages ont été enregistrés dans les structures des urgences, soit un volume à rapprocher du volume annuel d'activité des services d'urgences décrit par la statistique annuelle des établissements de santé (SAE) collectée par la DREES et dont le volume annuel s'établit en 2012 à 18,7 millions de passages, soit une estimation journalière moyenne de 50000 ;

- neuf personnes sur dix ont été prises en charge dans des points d'accueil d'urgences générales. Pour les enfants de 
moins de 16 ans, quatre passages sur dix ont eu lieu dans des points d'accueil d'urgences pédiatriques ;

- près de huit patients sur dix ont été pris en charge dans un établissement public, $13 \%$ dans un établissement privé à but lucratif et $6 \%$ dans un établissement privé à but non lucratif.

Dès à présent, des analyses plus fines confirment tantôt des observations de terrain ou corrigent des « fausses certitudes » sur le spectre du monde de l'urgence. On peut ainsi souligner que $32 \%$ des patients entreprennent des démarches dans les 24 heures avant leur passage aux urgences en contactant plus de trois fois sur quatre un médecin ou un pharmacien, et en joignant le SAMU pour $10 \%$ d'entre eux. On relève aussi que la moitié des patients restent moins de deux heures aux urgences, hormis ceux maintenus en observation. Les nourrissons ( 2 pour 1000 pour les enfants de moins d'un an) et les personnes âgées de plus de 75 ans ( $12 \%$ des passages pour $10 \%$ de la population générale) ou de plus de 85 ans ( $5 \%$ des passages pour $4 \%$ de la population générale) ont le recours aux urgences le plus élevé.

Cette enquête s'inscrit donc pleinement en complément utile aux données déjà collectées en routine, telles les informations recueillies dans le cadre de la Statistique annuelle des établissements de santé (SAE), du Programme de médicalisation du système d'information (PMSI), du résumé de passage aux urgences (RPU). Cette description s'appuie également sur des informations qualitatives issues d'une série d'entretiens réalisés en 2012 auprès de 110 professionnels des urgences, dans le cadre de la préparation de l'enquête nationale du 11 juin $2013[7,8]$.

Sous la houlette de Carlos El Khoury, la commission qualité de la SFMU travaille d'arrache-pied depuis plus de quatre mois pour faire de ce travail de recherche épidémiologique une base de données utile aux urgentistes et aux tutelles pour prendre les meilleures décisions possibles dans l'avenir. Une des réflexions menées parmi d'autres cherche à remplacer la classification clinique des malades aux urgences (CCMU), classification a posteriori, par un autre outil caractérisant au mieux, dès la première évaluation de chaque patient aux urgences, l'importance de la prise en charge que nécessite sa pathologie, depuis la simple consultation jusqu'aux soins de réanimation. Nul doute que les fruits de ces travaux et bien d'autres réflexions feront l'objet de communications au congrès Urgences 2015 et seront reproduits dans notre journal scientifique.

\section{Références}

1. Baubeau D, Carrasco V (2003) Motifs et trajectoire de recours aux urgences hospitalières, Études et Résultats. DREES, ${ }^{\circ} 215$, janvier

2. Carrasco V, Baubeau D (2003) Les usagers des urgences. Premiers résultats d'une enquête nationale. Études et Résultats DREES. $n^{\circ}$ 212, janvier

3. DREES. Recherche, études, évaluation et statistiques. www.drees. sante.gouv.fr, rubrique "Enquêtes, établissements de santé, sociaux et médicosociaux ». Consulté le 16 octobre 2014

4. DREES. Enquête nationale sur les structures des urgences hospitalières, juin 2013. http://www.drees.sante.gouv.fr/enquete-nationalesur-les-structures-des-urgences, $11113 . \mathrm{html}$. Consulté le 16 octobre 2014

5. Boisguérin B, Valdelièvre H (2014) Urgences : la moitié des patients restent moins de deux heures, hormis ceux maintenus en observation. Études et résultats. DREES, $\mathrm{n}^{\circ} 889$, juillet

6. Les points d'accueil des urgences hospitalières en 2013 : des organisations différentes entre établissements publics et privés. Études et Résultats. DREES (à paraître)

7. Vuagnat A (2014) Les urgences hospitalières, qu'en sait-on ? Le panorama des établissements de santé - édition 2013. DREES, p. $11-28$

8. La médecine d'urgence. Le panorama des établissements de santé - édition 2013. DREES, p. 136-7 